\title{
5G Mobile Communication Development Trend and Several Key Technologies
}

\author{
Liping Zheng \\ College of Optoelectronic and Communication Engineering, Yunnan Open University,Kunming, Yunnan Province, 650500, China
}

\begin{abstract}
The overall performance of $5 \mathrm{G}$ mobile communication network has made great progress compared with $4 \mathrm{G}$ network. In order to ensure the expected utility of $5 \mathrm{G}$ communication system in practical application, this paper will focus on the future development trend of this communication system and its research on key technologies involved in the application process to provide theoretical support to relevant practitioners.
\end{abstract}

\section{Introduction}

With the continuous development of China's society, people have put forward higher requirements for the security and transmission efficiency of mobile communication networks. The $4 \mathrm{G}$ mobile communication network obviously cannot fully meet the above requirements, and $5 \mathrm{G}$ mobile communication has emerged as the times require. In order to ensure that the $5 \mathrm{G}$ mobile communication system can be put into use in China as soon as possible, it is very necessary to study the key technologies involved in the application process. This paper mainly focuses on wireless transmission technology, wireless network technology and soft base station. On the other hand, the future development trend of $5 \mathrm{G}$ mobile communication has also received a great deal of attention. This article will discuss the needs of users in virtual reality, high-definition video, and Internet of Things.

\section{5G Mobile Communication Overview}

By definition, 5G mobile communication mainly refers to the fifth generation mobile communication system. Compared with $4 \mathrm{G}$ network, $5 \mathrm{G}$ mobile communication network has a great improvement in coverage, system security, user experience, etc. [1], At the same time, the $5 \mathrm{G}$ mobile communication system itself has very strong flexibility and can be automatically adjusted. Under the background of rapid development of various technologies and different industries, 5G mobile communication systems can still cope with such trends. Combined with the above content, 5G mobile communication will completely replace $4 \mathrm{G}$ mobile communication into the mainstream communication network in the future, so as to ensure that the different needs of different users can be better satisfied.
Combined with the status quo, various countries have regarded the research on $5 \mathrm{G}$ mobile communication and related technologies as the main research content, and with the continuous development of Internet technology, the combination of mobile communication technology and the Internet will inevitably become closer. Based on the $4 \mathrm{G}$ network, $5 \mathrm{G}$ mobile communication will be able to better provide users with better services through the Internet platform and wireless transmission technology. In addition, through the introduction of the new architecture, the throughput capacity of existing intelligent systems will be expanded by more than 25 times. The intelligent demand of various industries in the social development process will be able to be supported by $5 \mathrm{G}$ communication technology and get a better implementation.

\section{Analysis of the Development Trend of 5G Mobile Communication}

\subsection{Virtual Reality Aspects}

In recent years, virtual reality and related technologies have received extensive attention in the society. By definition, virtual reality mainly combines visual and auditory, and then simulates certain scenes for users to ensure that users can immerse them. With the increasing use of virtual reality technology in games, interactions, etc., users have put forward higher requirements for the experience gained from virtual reality. With the support of $5 \mathrm{G}$ technology, users' interaction experience between virtual and real world will be greatly improved.

\subsection{HD Video}

With the continuous development of computer technology and network technology, users have put forward higher requirements for video clarity. There are 
also a large number of high-definition and ultra-clear devices on the market. For these devices, the transmission speed and transmission quality are the main factors that determine whether these devices can provide the corresponding clarity [2]. In this regard, the transmission speed of $5 \mathrm{G}$ mobile communication is very fast, generally reaching Gigabit. Under such a background, HD video will be transmitted under unrestricted conditions, ensuring that users can access anytime, anywhere can watch HD videos.

\subsection{Smart City}

The concept of a smart city is no stranger to people. Through the construction of a smart city, people's daily production and living activities will be more convenient, and the effectiveness of local government management work can be improved. Combined with the application effect of the smart city at this stage, since this system needs to cope with the needs of thousands of users in a city, the throughput and transmission rate of the mobile communication network itself must meet the requirements, and then in the future development process. Ensure that the construction of a smart city can achieve the desired results.

\subsection{Internet of Things}

Combined with the status quo, the Internet of Things has been applied to a certain extent in all walks of life in China, and has facilitated the development of related work, but as the scope of the Internet of Things continues to increase, the network included in the Internet of Things the object will naturally become more. This trend means that with the continuous development of the Internet of Things and related technologies, the Internet of Things system will face more communication and security problems in the actual application process. The combination of $5 \mathrm{G}$ mobile communication network and Internet of Things is the main way to avoid the above problems. With the support of $5 \mathrm{G}$ mobile communication technology, all kinds of information in the Internet of Things will be processed in a wireless and high-speed way [3], information problems arising during transmission and interaction can naturally be well prevented.

\subsection{User Experience}

In addition to meeting the needs of users in communications, data transmission, etc., 5G mobile communications must be able to provide a better user experience on the original basis. On the other hand, under the experience-based service mode, the user's recognition of mobile communication will be better improved. Therefore, in the future development process, research on $5 \mathrm{G}$ mobile communication and related technologies must be able to put the user experience in an important place to consider.

\section{Research on Key Technologies of 5G Mobile Communication}

\subsection{Wireless Transmission Technology}

(1) Massive MIMO technology. MIMO is a multi-input and multi-output technology. This technology mainly uses multiple transmit and receive antennas at the transmitting end and the receiving end. The signal is transmitted through multiple transmit and receive antennas. This transmission mode can be used in spectrum resources. In the case of constant change, multiple transmissions and multiple receptions are realized, thereby improving the communication quality. Figure 1 is a block diagram of the MIMO technology. In the practical application process, massive MIMO has the following two advantages: First, with the support of this technology, different users will be able to communicate at the same time without restriction, and the spectrum efficiency will also increase through the increase of base station density. With the increase, the communication demand after the increase in the number of system users can naturally be better satisfied. Secondly, massive MIMO technology can concentrate the beam in a very narrow area, and the interference in the signal transmission process is relatively small, thus providing users with better quality information transmission services.

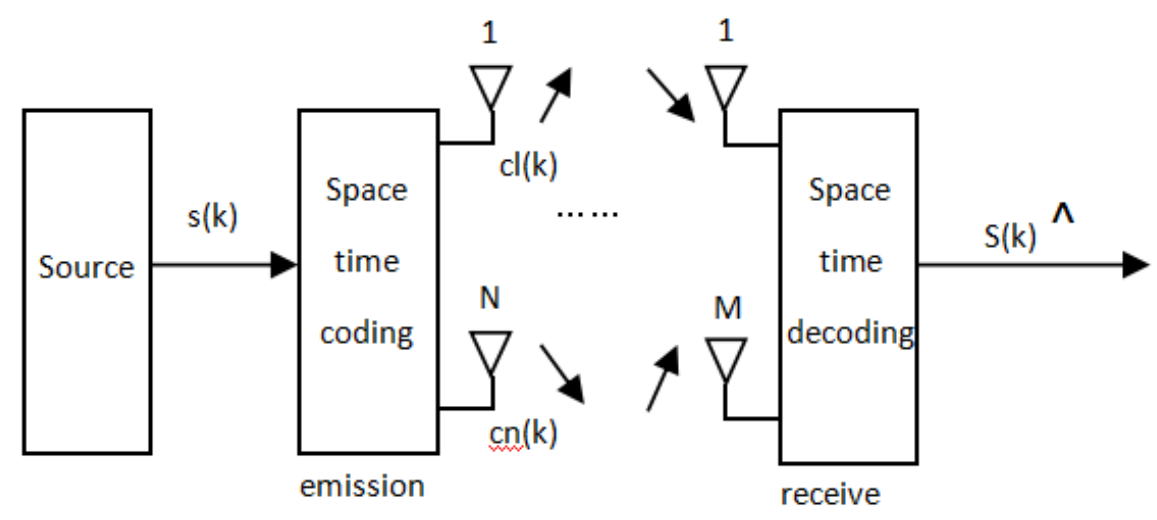

Figure 1 MIMO technology block diagram

(2) Multi-carrier technology based on filter banks.

The application of multi-carrier technology in wireless 
communication systems can effectively improve the spectrum efficiency and at the same time resist some multipath fading phenomena. However, in practical applications, multi-carrier technology requires that each sub-carrier must have the same bandwidth during the operation. These subcarriers also need to remain orthogonal. In this context, the application of multi-carrier technology actually has many boundaries and is less flexible. On this basis, the filter bank based multi-carrier technology solves the above problems well. This technology does not require orthogonality between carriers in the actual application process. Therefore, through the application of this technology, the bandwidth of each carrier can be independently set, and the overall flexibility of the system is greatly improved. Combined with these characteristics, the filter bank multi-carrier technology has been applied to a certain extent in radar signal processing, signal processing, etc. In the process of continuous development of $5 \mathrm{G}$ mobile communication networks, this technology can also be very good for control small parts in some systems.[4].

\subsection{Wireless Network Technology}

(1) Ultra-intensive heterogeneous network technology. The application of ultra-dense network heterogeneous technology in $5 \mathrm{G}$ system has the following advantages: First, because the network is relatively dense in this technology, the distance between the network node and the terminal is also closer, in such a background. Under the above, the spectrum efficiency can be effectively improved on the original basis, thereby meeting the requirements of the transmission rate of users in the system. Secondly, through the application of this technology, due to the increase of network density, the distance between nodes will inevitably decrease accordingly. With the increase of network density, the capacity of the whole system can be greatly improved, thus satisfying $5 \mathrm{G}$. The trend of increasing the number of system users and the number of terminals. The application of ultra-dense network heterogeneous technology in $5 \mathrm{G}$ systems still needs to be improved in the following aspects: due to the reduction of the distance between nodes in the system, the probability of problems in the system running process will naturally show a certain upward trend, in order to avoid the impact of this feature on the normal operation of $5 \mathrm{G}$ systems, relevant technicians can use wired backhaul to better save resources and simplify procedures [5].

(2) Self-organizing network technology. Self-organizing network is a network combining mobile communication and computer network. Under the function of this technology, user terminals can move freely within the network while maintaining communication. As people's demand for free communication grows, self-organization The network has been applied to a certain extent in military, civilian, commercial and other aspects. For 5G mobile communication technology, $5 \mathrm{G}$ is a heterogeneous network with co-existence of converged and coordinated multi-systems. With the continuous changes of services, time and space, 5G mobile communication networks will also undergo certain changes. In order to better adapt to these dynamic changes, technicians must be able to achieve this through the effective deployment of network deployment work. At the same time, in order to ensure the smoothness of communication when the user moves, the technician should be able to optimize the selection of the target through the dual connection form to ensure that the information transmission under the self-organizing network can meet the user's needs.

(3) Content distribution network. The content distribution network mainly refers to adding a new network architecture to the existing network, and then using this architecture to publish the network content to the "edge" of the network closest to the user, the user can obtain the required content nearby, thereby solving the network congestion. Status, adjust the response speed to user access. For the 5G mobile communication network, as the number of users accesses increases, the network bandwidth will inevitably support such requirements, and eventually the overall response speed of the system is too slow, and the user needs are not met. With the support of related technologies of content distribution network, the above problems can be effectively solved, and the impact of small network bandwidth, large user access, and uneven distribution of network points is technically avoided. For the implementation of the content distribution network in the $5 \mathrm{G}$ system, the technician can adopt the cache server and distribute the servers as much as possible to the area where the user access requirements are concentrated. Combined with the network traffic and the connection between the nodes, the user information will be directed to the nearest service point, and then the corresponding information can be obtained through this service point. The slow response speed of the website can be effectively solved.

\subsection{Soft Base Station}

By definition, a soft base station mainly refers to a radio frequency unit connected to a remote large-capacity baseband processing resource pool through a fiber or other digital transmission medium in a coverage area, thereby sharing baseband processing resources among the radio frequency units, and control the clock unit, operation and maintenance platform, etc., and finally realize the base station system covering the surrounding neighboring areas. By merging $5 \mathrm{G}$ soft base stations into the same hardware platform, the $5 \mathrm{G}$ system will be able to adapt to more software requirements, thereby breaking the traditional way of constructing the network and ensuring that users can communicate flexibly in the system. In the actual application process, the $5 \mathrm{G}$ soft base station mainly has the following features:

(1) Distributed coverage. The main unit of the soft base station is mainly connected to the substation through the digital transmission device. Therefore, the distance between the substation and the main unit is relatively small. In the initial stage of the $5 \mathrm{G}$ system construction, the technician can pass the main substation which is pulled around the unit to form a continuous 
coverage of the large area. Under such a background, the coverage area of a $5 \mathrm{G}$ soft base station will be increased by several times to several tens of times compared with a conventional base station.

(2) Softer switching. In combination with the above, the soft base station substation and the main unit share the baseband resource pool. Therefore, in the actual application process, the substation can be regarded as a sector of the primary station, between different sectors between the same base station. Switch to a softer switch. With the support of this feature, the system capacity of 5G mobile communication networks will be greatly improved.

(3) Soft Planning. On the basis of the second content, if the substation can be regarded as the remote sector of the main unit, the substation in the system can also be regarded as the different logical base station of the main unit, on the basis of which The neighboring base stations uniformly plan the PN code and the carrier frequency plan, and the difficulty of network planning will be greatly reduced. Such an advantage is very beneficial in the application of the $5 \mathrm{G}$ system.

(4) Soft business capabilities. In the development of $5 \mathrm{G}$ mobile communication, the communication system necessarily needs to be improved with the change of related technologies and requirements. In this process, since the sub-station in the soft base station only exists as the radio remote end of the main unit. Therefore, the technician only needs to upgrade the main unit. Under such a background, the expansion of network upgrades and business upgrades will become easier and more convenient, greatly improving the actual application effect of $5 \mathrm{G}$ systems.

(5) Soft baseband resources. The baseband processing on the main unit side of the soft base station adopts the resource pool design. Therefore, the base station resource pool can be shared between the sub-station under different standards in the system and the sub-station with different frequencies in the same standard. Under the influence of this feature, the channel resource utilization rate in the $5 \mathrm{G}$ system can naturally be effectively improved. It is worth noting that the sharing of baseband resources between substations is dynamic. The main unit will allocate hardware resources according to the dynamic needs of different substations, that is, the baseband resources exhibit soft characteristics for substations.

\section{Conclusion}

In summary, this paper mainly analyzes the development trend and key technologies of $5 \mathrm{G}$ mobile communication, and the related technologies should further study these contents in the subsequent development process to ensure that $5 \mathrm{G}$ mobile communication can play its expectations in the actual application process to meet the communication needs of users.

\section{References}

1. Huang Wenchao. Discussion on the development trend of $5 \mathrm{G}$ mobile communication and related key technologies [J]. Electronic Test, 2017, (7).

2. Zhang Weimin, Zhao Xinxin. Analysis of key technologies and development trends of $5 \mathrm{G}$ mobile communication $[\mathrm{J}]$. Wireless Internet Technology, 2018, (13).

3. Fang Ruyi. Key Technologies and Analysis of 5G Mobile Communication Network [J]. Information Technology, 2017, (1).

4. Chen Jiaming. Key technical specifications and development of $5 \mathrm{G}$ mobile communication [J]. Telecommunications Engineering Technology and Standardization, 2018, (6).

5. Xu Guiping. Key Technologies and Processes of $5 \mathrm{G}$ Mobile Communication [J]. Technology Wind, 2018, (5). 